Retos, I0(19), 2020

\title{
Señalización y el éxito de las campañas de crowdfunding latinoamericano
}

\section{Signaling and success in campaigns of Latin-American crowdfunding}

Ing. Walter M. Sánchez Fontana graduado de la Universidad del Azuay (Ecuador) (mateosanchezf@es.uazuay.edu.ec) (https://orcid.org/0000-0001-5401-3729)

Econ. Luis B. Tonon Ordóñez es profesor e investigador de la Universidad del Azuay (Ecuador) (ltonon@uazuay.edu.ec) (http://orcid.org/0000-0003-2360-9911)

\section{Resumen}

Las oportunidades de financiamiento para emprendimientos en Latinoamérica son pocas, limitándose comúnmente al uso de instituciones tradicionales como la banca pública y privada, o incluso a recursos recaudados informalmente, ninguno de estos particularmente estructurado para emprendimientos de etapa temprana. Además, han surgido nuevos mecanismos de recaudación como el crowdfunding basados en recompensa. Sin embargo, este sufre en Latinoamérica de grandes porcentajes de fracaso y un crecimiento general desacelerado en comparación a otras regiones. Esta investigación empírica, pretende contribuir al entendimiento de los factores que hacen a una campaña exitosa desde la perspectiva de la teoría de señalización. Con base en la información recaudada de 21804 campañas de origen latinoamericano, extraídas de ocho países distintos, de las plataformas Catarse, Kickstarter e Idea.me. Los resultados indican que el uso de redes sociales, número de recompensas, material multimedia incluyendo imágenes, videos y GIF, así como esfuerzos para comunicarse con el cliente sea durante o después de culminado el plazo de recaudación, influyen positivamente al éxito de una campaña. Se determina también un uso marginal de todas estas señales, independientemente del país de origen de las campañas o su naturaleza. El mermado crecimiento de la escena de crowdfunding en la región se observa que está en parte afectada por la calidad inherente de los proyectos, donde hay mucho espacio por mejorar en referencia a las señales usadas.

\begin{abstract}
Funding opportunities for entrepreneurships in Latin America are few. Commonly being limited to the use of traditional platforms such as public and private banking, or even informal forms of recollecting recourses, none of this particularly fitted to support early stage entrepreneurship. Because of this, new collection mechanisms have emerged, such as reward-based crowdfunding. However, this mechanism presents large percentages of failure and diminished growth rates in comparison to other regions. This empirical research aims to contribute to the understanding of the factors that make a successful campaign from the perspective of signaling theory. Based on the information collected from 21804 Latin American campaigns, extracted from eight different countries, of the Catarse, Kickstarter and Idea.me platforms. The results indicate that the use of social networks, number of rewards, multimedia material such as images, videos and gifs, as well as efforts of communication with clients, it being during or after the funding phase; have a positive influence in the success of a campaign. Furthermore, all of these are seldom used by the majority of evaluated campaigns, independent of their country of origin or the nature of the campaign itself. The lessened growth of crowdfunding on the region is understood, is at least in part affected due to the inherent quality of the projects, where's a lot of room for improvement to be made.
\end{abstract}

\section{Palabras clave I keywords}

Crowdfunding, recompensa, señalización, extracción de datos, comercio en línea, asimetrías, financiamiento, emprendimiento.

Crowdfunding, reward, signaling, data extraction, e-commerce, asymmetries, funding, entrepreneurship.

Cómo citar: Sánchez Fontana, W. M., y Tonon Ordóñez, L. B. (2020). Señalización y el éxito de las campañas de Crowdfunding latinoamericano. Retos Revista de Ciencias de la Administración y Economía, 10(19), 99-116. https://doi.org/10.17163/ret.n19.2020.06 


\section{Introducción}

En toda economía en vías de desarrollo, el fomento de la inversión privada es una prioridad, sin embargo, una de difícil ejecución. Esto debido a varios factores, siendo el principal las complicaciones que presenta el incentivar la disposición al financiamiento por parte de entidades financieras tradicionales, que entendiblemente representan una alternativa de financiamiento muy cautelosa, que por su naturaleza misma filtran y, por consecuencia, dejan sin oportunidades a inversiones de mayor riesgo, como son los emprendimientos de etapa temprana.

En respuesta a estas dificultades, en los últimos años han surgido instrumentos que permiten el financiamiento de inversiones de mayor riesgo, algunas de estas fundamentándose en el internet como su plataforma. Este es el caso de crowdfunding, un esfuerzo por democratizar el poder de financiamiento de la persona común y distribuir el riesgo en una mayor cantidad de inversionistas.

El interés en avanzar la siguiente investigación nace del potencial que puede tener una herramienta como el crowdfunding en la escena de emprendimiento latinoamericano. El mismo que ya ha empezado a desarrollarse lentamente, sobre todo en sus modelos de menor complejidad, como el crowdfunding basado en recompensa, el mismo que funciona de forma similar a una página de comercio electrónico común, con la diferencia para el consumidor de pagar por adelantado por el producto y estar confiado en tan solo el prototipo del producto.

Para introducir este nuevo modelo de financiamiento en masa, es preciso entender los factores que influyen en hacerlo una alternativa más atractiva para el inversionista, que en este caso sería el consumidor común. En estos esfuerzos, los trabajos realizados hasta la fecha, sobre todo en mercados más experimentados como el estadounidense, han apuntado al estudio de la señalización de calidad en las plataformas web y en los proyectos, como uno de los mecanismos por los cuales impulsar este modelo de inversión.

Así, esta investigación se esfuerza por entender qué tan bien las plataformas y proyectos de las principales páginas de crowdfunding en Latinoamérica están implementando esfuerzos de señalización básicos, a fin de determinar qué tanto de la dificultad por levantar el porcentaje de éxito de los proyectos de crowdfunding en estas páginas se debe simplemente a la calidad de los proyectos presentados y qué tanto se debe a una general falta de cultura de consumo en línea de la región.

Si se considera el supuesto que mientras mejor es el uso de señalizaciones de calidad por parte de los proyectos, mayores serán sus probabilidades de recaudar exitosamente el dinero pedido. Esto será analizado y respondido dentro de la investigación, a través de la recolección de información del universo de proyectos de origen latinoamericano en tres de las plataformas más usadas en la zona, a través del uso de un robot de búsqueda desarrollado para este propósito.

\section{Revisión de literatura}

Un reto mayor que implica la creación de cualquier emprendimiento es la obtención de capital inicial para cimentar la puesta en marcha de una empresa. Tradicionalmente, de no poder autofinanciarse o apelar a lazos familiares, los emprendedores acuden 
al sector intermediario (principalmente a bancos comerciales) en búsqueda de préstamos a largo plazo (Berger \& Udell, 1995); o incluso en ciertos casos a modelos de inversiones ángeles e inversiones de capital de riesgo. Sin embargo, el primero tiene comúnmente considerable aversión a financiar estos proyectos, debido al alto riesgo que implican, además de que el pago de interés mensualizado puede resultar complejo, dada la volatilidad financiera que pueden tener en un inicio dichos proyectos. El segundo es poco común y puede requerir de mucha injerencia del inversor en las decisiones sobre el proyecto. Esto genera un agujero de oportunidades de financiamiento importante (Kunz, Bretschneider, Erler \& Leimeister, 2017), sobre todo para el impulso de nuevos proyectos, más aún si estos se tratasen de modelos de negocio con productos o en sectores experimentales (Mason \& Harrison, 2004).

Añadido a estas complicaciones, el levantamiento de fondos no es el fin de las complicaciones para un emprendimiento. Tras encontrar algún tipo de financiamiento sea interno o externo, el nuevo negocio deberá empezar a producir o desarrollar un servicio bajo su propia cuenta o bajo la tercerización de esta función; para más tarde tratar de venderlo directamente, o bajo un intermediario. Este método tradicional es caro y sobre todo riesgoso, dadas las fuertes suposiciones que se deben realizar sobre la real demanda del producto (Chakraborty \& Swinney, 2016), así como la sanidad general de sus flujos de efectivo para poder cubrir la deuda u obligaciones de inversionistas.

Sin embargo, el creciente uso de internet como un medio amistoso a variados tipos de transacciones económicas y financieras (Dehling, 2013), ha desencadenado en la creación de nuevas formas de financiamiento que acogen aquellas debilidades y sectores de la demanda financiera desatendidos por los formatos tradicionales. Estos nuevos modelos de financiamiento han resultado en conexiones poco comunes entre emprendedor e inversor, donde este último puede tomar distintas formas, como la de cocreador, consumidor, patrocinador, inversionista o simplemente prestamista (Davies \& Giovanetti, 2018). Lo que es consistente en todos ellos es la ausencia de un gran intermediario y, por el contrario se apela, por las características del medio en que se encuentra al libre acceso, en el que se estimula la accesibilidad y cantidad de inversionistas (Dehling, 2013; Agrawal, Catalini \& Goldfarb, 2013; Lambert \& Schwienbacher, 2010). El más importante de estos mecanismos en línea es llamado crowdfunding.

Crowdfunding es relativamente un nuevo modelo financiero el cual democratiza el poder de financiamiento, es decir, es usualmente de libre acceso al ciudadano común y es definible como "los esfuerzos de emprendedores y grupos sin fines de lucro a financiar sus proyectos al atraer pequeñas contribuciones de grandes números de individuos por medio de plataformas en internet" (Mollick, 2013, p. 2). Por lo tanto, la dinámica por la cual funciona el crowdfunding está compuesta de tres actores (Figura 1): los emprendedores, responsables del proyecto; la plataforma en línea, como vehículo para la exposición y transacción; y los inversores, que pueden llegar a tener varias formas, dependiendo de que se le ofrezca a cambio. 


\section{Figura 1. Modelo General de crowdfunding}

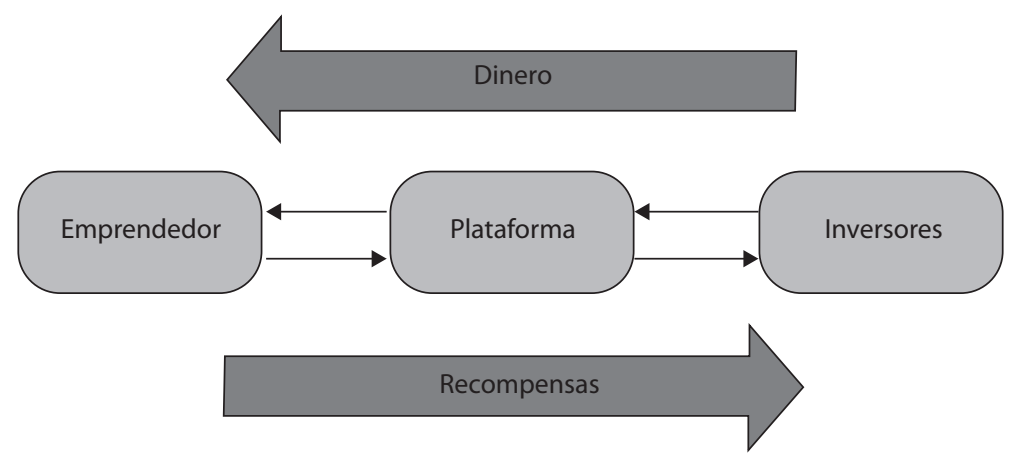

Fuente: Davies y Giovannetti, 2018

Este modelo es particularmente útil para emprendimientos y pequeñas empresas, dado que, al tratarse de tan pequeñas contribuciones de dinero, sus inversores experimentan poca aversión al riesgo, además sus intermediarios son las plataformas que gestionan el intercambio de dichos fondos y estas subsisten de comisiones que fluctúan comúnmente entre el 3\% y $8 \%$, todas funcionando en línea (Wells et al., 2011).

Existen en concreto cuatro modelos por los cuales los inversionistas financian en crowdfunding (Agrawal et al., 2012; Banco Mundial, 2013; Belleflamme, Lambert \& Schwienbacher, 2013); donde la diferencia yace en la forma en la que se recompensa al inversor. Esto tiene grandes implicaciones en cómo funciona cada uno de ellos. Estos son por donaciones, el más sencillo de todos dado que no comprende una recompensa mayor a cambio, usado para causas sociales, donde sólo subraya un sentido de altruismo en sus participantes; por préstamos, donde este actúa bajo una promesa de pago a quien financia y también apela a un sentido de altruismo o interés individual del participante; por recompensas, el que bajo un mayor nivel de complejidad, es el financiamiento a cambio de un producto o servicio específico; y por adquisición de acciones, el más complejo de todos y que progresivamente va tomando mayor tracción (Massolution, 2015), que comprende la adquisición de una porción de la empresa. Cada uno de estos tiene sus propias dinámicas y teorías que respaldan el éxito o fracaso de un proyecto.

El estudio propuesto, se centra específicamente en el modelo basado en recompensa, el cual funciona como la promesa de venta (preventa) de un producto o servicio (Chakraborty \& Swinney, 2016); el cual, el emprendedor desea producir, en caso de que el financiamiento 'objetivo' para poner en marcha dicho negocio, se cumpliese (Agrawal et al., 2013).

Se elige este modelo para el estudio, dado que este es el predominante, más aún en Latinoamérica donde más de las tres cuartas partes de visitas a sitios de crowdfunding, son a plataformas de este tipo (Rentería, 2016); y es también el modelo 
del cual existe mayor accesibilidad de información (Dehling, 2013; Banco Mundial, 2013). Así también su nivel de complejidad medio ha permitido su introducción en Latinoamérica, dado que es muy accesible al público, al comportarse mayormente como una dinámica de comercio en línea común.

Crowdfunding en todas sus formas ha pasado ya de ser un fenómeno prometedor a una estructura consolidada y de alto crecimiento. Donde en 2015 a nivel mundial se recolectó 34.4 mil millones de dólares, con crecimientos de $270 \%$ y $200 \%$ en sus años anteriores (Massolution, 2015) y se espera que para 2020 alcance recaudar un estimado de 90 mil millones de dólares (Forbes, 2015). El fenómeno de las plataformas de crowdfunding de todo tipo se catapultó en los últimos años (Banco Mundial, 2013), primordialmente en Estados Unidos, después de la introducción de normativas impuestas para la aplicación más libre de todos los modelos de crowdfunding en el JOBS Act (Dehling, 2013). Estas nuevas legislaciones pavimentaron el camino para la réplica y expansión de este modelo de financiamiento por Europa, y más recientemente por partes de Latinoamérica.

Tan solo en Kickstarter, la principal plataforma de crowdfunding basado en recompensa, desde su fundación en 2008 ha levantado 3,9 mil millones de dólares a través de la materialización de 420 mil proyectos. De estos proyectos, los que mayor tracción han tenido, han recolectado entre 13 y 20 millones de dólares. Además de sus números, estas plataformas han permitido la comercialización de nuevas tecnologías como relojes, estantería y conexiones eléctricas inteligentes, telas repelentes al agua, nuevos formatos de apicultura y un sinnúmero de gadgets novedosos.

La escena de crowdfunding en Latinoamérica es, en general, incipiente con relación al avance en otros continentes. En 2014 se financió en Latinoamérica un total de 57.2 millones de dólares, con un crecimiento anual de 167\%, porcentaje que ha ido disminuyendo con los años, y es un nivel de crecimiento cercano al que manejan los mercados más avanzados como el estadounidense o europeo que rondan el $140 \%$. De hecho, el mercado que parece significativamente más prometedor es el asiático con crecimientos anuales sobre el 300\% y un financiamiento de 3.4 mil millones en 2014 (Massolution, 2015).

De todas formas, las cifras son igualmente desalentadoras. De todo el financiamiento levantado por crowdfunding a nivel mundial en 2013, tan solo un $0.4 \%$ de este fue generado en Latinoamérica. Esto pudiera deberse a tres distintos contingentes. Primero pudiese estar provocado por la falta de plataformas latinoamericanas, donde en 2014 solo existían 50 y a un crecimiento muy pequeño; segundo, pudiese deberse a la falta de cultura de consumo de este tipo en la región; o por último a que los proyectos lanzados no cumplen con estándares de calidad suficientes, lo cual iría congeniado con la falta de tracción de proyectos de origen latino incluso en plataformas internacionales como Kickstarter (Massolution, 2015).

Aunque el crecimiento del crowdfunding latinoamericano no vaya a la par con respecto a otras regiones. los volúmenes aún son representativos, y sus tasas de crecimiento son altas en comparación a otras alternativas de financiamiento; lo cual es prometedor si se considera que este es un fenómeno muy concentrado en pocos países y que, por tanto, tiene potencial de crecimiento significativo. 
La escena de crowdfunding latinoamericano, como el desarrollo de este no ha sido parejo en los distintos países de la región. Por el contrario, la actividad está sumamente concentrada en Brasil, donde en 2015, el porcentaje de visitas diarias dirigidas a plataformas brasileñas era el $42.2 \%$ de todo el mercado sudamericano (Rentería, 2016; Kickante, 2018); así mismo, los países con actividades medias son Argentina, Chile y México, este último a pesar de no poseer una plataforma nacional fuerte (Nafin México, 2017); y el resto de los países latinos están aún en un proceso de iniciación.

En cuanto refiere a plataformas, no es sorpresa que estas a su vez sean muy concentradas y pocas conglomeran la mayoría de las visitas. Las principales son Catarse, Idea.me, Vakinha, Prestadero, Panal de ideas y Broota (Rentería, 2016). De las cuales todas son plataformas basadas en recompensa a excepción de Broota, identificando una clara tendencia. Broota es la única plataforma prominente de crowdfunding basado en inversión, lo cual sugiere todavía la infantilidad del mercado latino en términos legales y culturales frente a este fenómeno.

Crowdfunding es un formato muy atípico de financiamiento, de modo que en un inicio era de principal interés académico entender cuáles son las bases que hacen a una campaña de crowdfunding exitosa (entendiéndose por exitosa, a aquellas campañas que recolectan el $100 \%$ o más de la meta de financiamiento previamente definida), y saber si modelos anteriores que predecían el financiamiento tradicional de proyectos en etapas tempranas eran válidos dentro de las dinámicas de crowdfunding (Mollick, 2013). La literatura que explica los comportamientos del mercado crediticio, sobre todo aquel en etapa temprana, es uno caracterizado por asimetrías de información. Esto entendiéndose como formas de transacción en las que existen altos grados de incertidumbre sobre la confiabilidad de que el otro actor pueda cumplir con su parte del trato (Connelly, Certo, Ireland \& Reutzel, 2011; Spence, 1973). En el caso de las ofertas financieras tradicionales esto se ve reflejado en una fuerte aversión al financiamiento de emprendimientos por la falta de información confiable sobre el prestatario o su proyecto (Davies \& Giovannetti, 2018).

La influencia de la asimetría de información en crowdfunding basado en recompensa no es menor, puesto que esta aqueja a las transacciones de este fenómeno por dos distintos frentes; primero por ser un modelo crediticio y segundo por ser en su naturaleza muy parecido a una transacción en un mercado electrónico, el cual a su vez también está influenciado por asimetrías de información (Kunz et al., 2017).

Claramente esta es la debilidad del crowdfunding basado en recompensa, pues comprende una transacción en la que se requiere mucha confianza, puesto que el comprador/inversionista no tiene seguridad de que el producto o servicio que está adquiriendo sea de hecho el exactamente ofrecido. Esta inseguridad es característica de las compras en línea, puesto que existen solo formas limitadas de saber si el producto luce y funciona como se está vendiendo (Dehling, 2013; Kunz et al., 2017; Kim, Buffart \& Croidieu, 2016; Martens, Jennings, \& Jennings, 2007). Por si esto no fuese suficientemente limitante, la compra en crowdfunding basado en recompensa en su mayoría no es inmediata, como lo sería en una compra en línea, sino que se estaría entregando el dinero tan solo bajo la promesa de que el producto será fabricado y enviado, luego de un espacio de tiempo, a las manos del consumidor. Esto, por lo 
tanto, hace al financiamiento a través de crowdfunding basado en recompensa uno muy peculiar y complicado de materializar (Belleflamme et al., 2012).

Ante estas claras limitantes, existen medidas que pueden ser tomadas en orden a mitigar los impedimentos que la asimetría de información impone. Estas están representadas bajo la teoría de la señalización (Mollick, 2013; Etter, Glossglauser \& Thiran, 2013; Chakraborty \& Swinney, 2016; Kunz et al., 2017); que se explica como un conjunto de 'señales' las cuales son “atributos que presenta un individuo dentro de un mercado, los cuales, intencionalmente o no, alteran las creencias y expresan información al resto de individuos en el mercado" (Spence, 1973, p. 263); es decir, en su aplicación puntual a crowdfunding son conjuntos de información que sugieren al consumidor la calidad y confiabilidad del producto o servicio (Cardon, Sudek, Mitteness, 2009). Estos son comúnmente usados en comercio electrónico para el mismo propósito, y se materializan bajo la particular presentación del producto, formatos de página y todo el material multimedia que lo envuelve, botones de pago y cualquier otra señal que sugiera que el vendedor es fiable (Jiang \& Benbasat, 2007).

En el caso particular de crowdfunding basado en recompensa, la señalización funciona de un modo muy similar a como lo hace en comercio electrónico. Sin embargo, la diferencia yace en que las plataformas de crowdfunding usan formatos comunes de presentación en orden a estandarizar y dar todas las herramientas a los emprendedores para presentar bien sus proyectos. Por ende, bajo este formato estándar de presentación de las campañas, ejemplos de señales de calidad pudiesen ser: el video que expone el prototipo (del bien o servicio), el número y formas de recompensas, estas entendiéndose como el portafolio de la campaña, el número de actualizaciones que hace a su página, la cantidad de comentarios que deja la gente y la respuesta a dichos comentarios, tracción que genere en redes, etc. (Belleflamme, 2012; Mollick, 2013; Kunz et al., 2017; Lynn, 1991; Nahapiet \& Ghoshal, 1998).

\section{Materiales y método}

Para esta investigación se ha evaluado los atributos medibles que hacen a una campaña de crowdfunding tener mayor calidad, bajo la perspectiva de la teoría de señalización. Además, bajo esta información también evaluar la existencia o no de distinciones significativas entre el país de origen de estos proyectos y su calidad (desempeño en su uso de señales); en orden a entender si la señalización y, por ende, la inherente calidad de los proyectos, tiene efecto en cómo se desarrolla el crowdfunding tan desigualmente por la región.

De dicho modo, se ha estipulado una revisión básica de señales, compuestas por las siguientes (Tabla 1), las que a su vez se han subdividido por su costo relativo de aplicación y en qué momento de la campaña es aplicado. Se ha utilizado el mismo formato de presentación usado por Kunz et al. el que a su vez está fundamentado en un formato utilizado para la señalización en páginas de comercio electrónico. 
Tabla 1. Señales a usar bajo formato Mavlova et al.

\begin{tabular}{|c|c|c|}
\hline & $\begin{array}{l}\text { Previo al inicio } \\
\text { de la campaña }\end{array}$ & Durante la campaña \\
\hline \multirow{2}{*}{ Bajo Costo } & \multirow{2}{*}{$\begin{array}{l}\text { Uso de cuentas en redes sociales } \\
\text { (Facebook, Instagram y Twitter }\end{array}$} & Número de comentarios \\
\hline & & Actualizaciones \\
\hline \multirow{3}{*}{ Alto Costo } & Cantidad de imágenes & Elegida entre favoritos (staff picked) \\
\hline & $\begin{array}{l}\text { Cantidad de videos } \\
\text { Página web propia }\end{array}$ & \\
\hline & Número de recompensas & \\
\hline
\end{tabular}

Fuente: Kunz et al., 2017

Para la investigación se recolectaron los datos pertinentes al universo de campañas de origen latino, de las plataformas Catarse, Kickstarter e Idea.me. Siendo estas tres de las plataformas de principal uso en la mayoría de los países de la región. Tan solo se han filtrado campañas de origen estadounidense o con características fuera de lo que se consideraría una campaña de crowdfunding basado en recompensa, es decir, se han obviado campañas de donación y causas sociales.

Aplicados todos estos filtros se consolida un universo extraíble de 21804 proyectos. De estos un 74.54\% pertenece a la plataforma Catarse, es decir 16253 proyectos; segundo un $13.22 \%$ para Kickstarter que se traduce en 2882 proyectos; y por último, un $12.24 \%$ para Idea.me que suma los restantes 2669 proyectos.

Estas páginas web al no tener un API, ${ }^{1}$ obligan a que la extracción de estas cantidades de datos, deban ser llevados a cabo por medio de un programa de Web Crawler. ${ }^{2}$ Estos pueden ser entendidos como "programas de búsqueda capaces de iterativa y automáticamente descargar páginas web completas, extrayendo sus URLs y recabando información dentro de ellas al detalle" (Thelwall, 2001, p. 319). Para ello se ha desarrollado uno para este propósito y se han extraído las variables expuestas en la Tabla 1, así como variables descriptivas complementarias, en orden a poder presentar la información que incluyen nombre de la campaña, categoría, país de origen, monto meta y monto recaudado.

Para poder constatar la relación entre las señales utilizadas por los proyectos y el éxito de estas, es decir, si es que la campaña logró recaudar el dinero que inicialmente se propuso como meta, se debe determinar su relación probabilística por el uso de una regresión logística binaria, como sugiere la literatura (Courtney, Dutta \& Li, 2017; Mollick, 2013; Kunz et al., 2017; Davies \& Giovannetti, 2018). Donde se utiliza como variable dependiente a una variable ficticia o dummy que indica el éxito

1 Interfaces propias de las empresas, las cuales permiten a cualquier usuario extraer metadatos de sus URLs.

2 Softwares gratuitos de extracción de datos, los que pueden colectar cualquier requisito (atributo en una página) que se le indique. 
(1) o fracaso (0) de una campaña, está a su vez controlada por las variables independientes que se evalúen (señales), tanto de forma separada como en combinaciones.

Para realizar este análisis se utilizará el programa estadístico SPSS, la cual dispone de los instrumentos necesarios para la realización de una investigación de estas características (Kunz et al., 2017).

Este tipo de análisis es más fácilmente detallado por su distintiva forma gráfica en forma de s, que trabaja en un rango de 0 a 1, como se puede observar en la figura 2 .

Figura 2. Diferencias entre regresión lineal y regresión logística

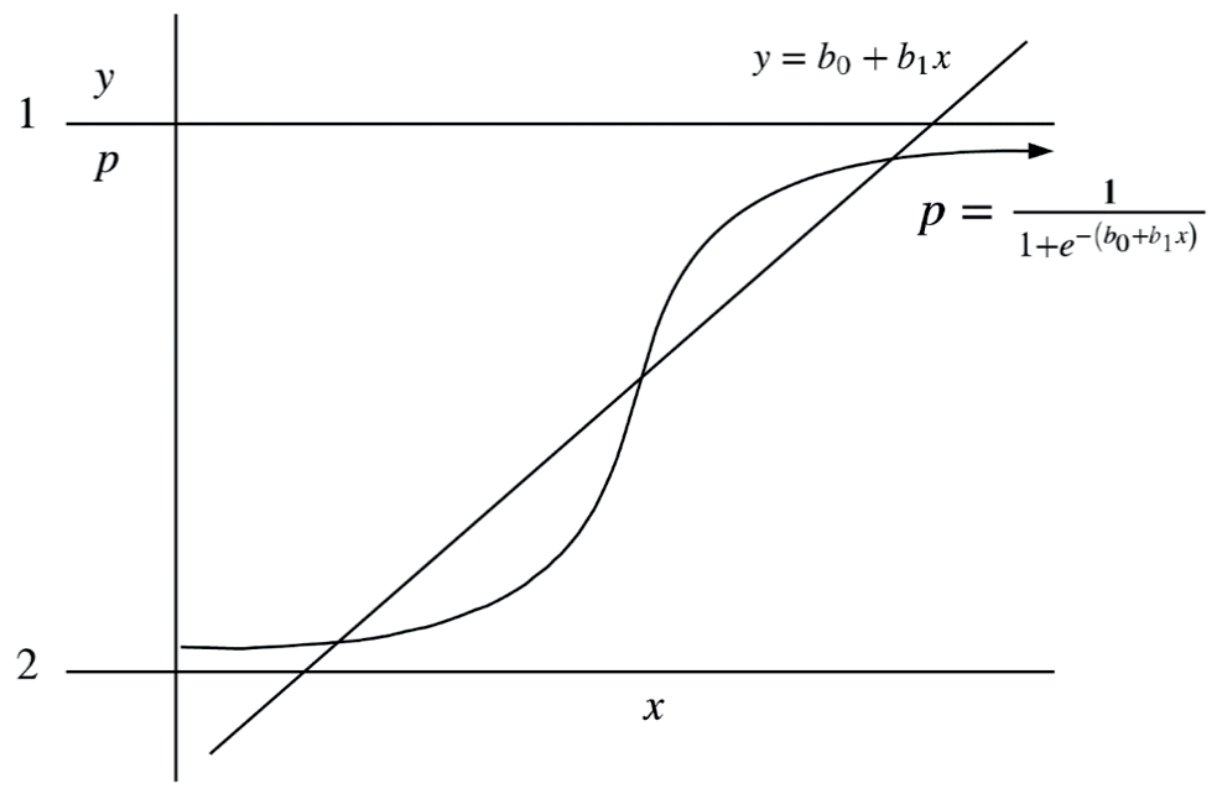

Fuente: saedsayad.com, n.f.

A través de este tipo de análisis se busca encontrar bajo el uso de varios modelos, combinaciones de variables independientes que prueben los mayores valores predictivos sobre el éxito de una campaña, mientras que estos tengan la menor cantidad de variables, para determinar los efectos de forma más armoniosa (Tranmer \& Elliot, 2005).

En un inicio, la regresión logística binaria pide una evaluación bi-variante, donde se evaluará el efecto singular de cada variable independiente o señal y su significancia en el contraste de su Chi cuadrado (Canela, Lora \& Estrella, 2011). Para reforzar el entendimiento de estas relaciones, se puede generar primero tablas de contingencia que evalúan el contraste de la hipótesis y una medida de asociación (Walsh, 1987). 


\section{Resultados}

A la fecha del 26 de noviembre de 2018, se pudo recabar 21803 proyectos, 6586 de estos fueron exitosos, es decir un $30.21 \%$.

Como sería de esperarse, gran parte del universo se concentra en proyectos de origen brasileño, comprendiendo un $75.53 \%$, primordialmente en Catarse, pero también por su presencia en Idea.me; luego le sigue México con un 11.36\% y Argentina con $8.76 \%$; la suma de las demás procedencias agrupa cerca de un 5\%. Así demostrándose el desequilibrio existente en el desarrollo de crowdfunding en la región, y las plataformas que las representan, como es claramente observable en la figura 3.

\section{Figura 3. Clasificación de todos los proyectos por su país de origen}

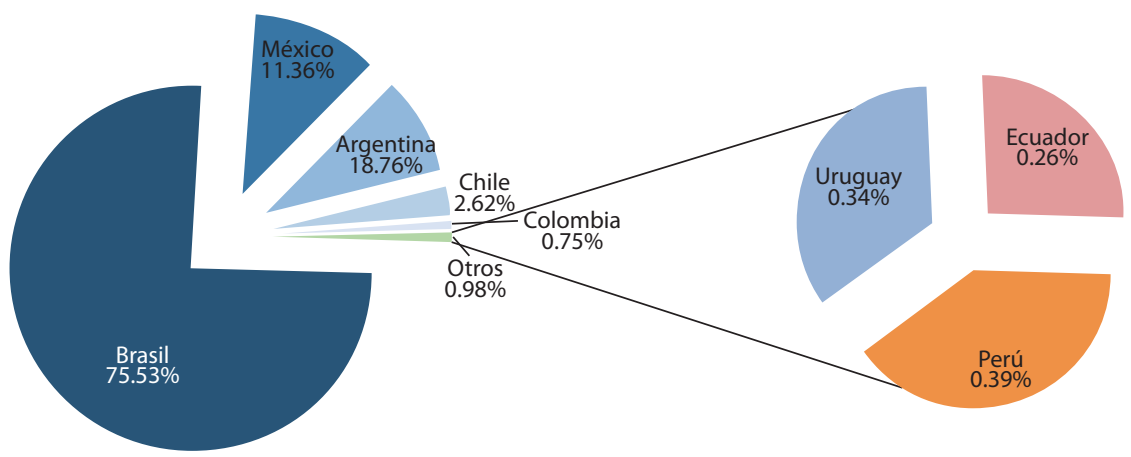

En cuanto al desempeño de los proyectos, es preciso entender el número de inversionistas que pueden atraer, la tendencia es muy clara, la moda general de los proyectos es de cero inversionista y la mayoría de los inversionistas se acumulan en muy pocos proyectos, haciendo de la escena de crowdfunding una muy desigual, donde los proyectos que fracasan, lo hacen rotundamente y cuales son exitosos lo logran por muy poco (Mollick, 2013); después de todo tan solo 2843 proyectos, es decir, el $13.20 \%$, llegan a tener más de 100 inversionistas y las probabilidades van disminuyendo significativamente; 317 proyectos, es decir, el 1.45\% llega a tener más de 500 inversionistas

El uso de páginas web propias para generar tráfico y valor fuera de la plataforma sigue un patrón no del todo homogéneo a través de las plataformas, siendo mucho mayor su uso en Idea.me, aproximándose al 57\%. Sin embargo, la media se da en un $32.60 \%$ de uso, muy aproximado al $28.90 \%$ y $29.84 \%$ visto en las plataformas Catarse y Kickstarter, respectivamente. Es concluyente que su uso es representativo, pero no generalizado, por lo que puede constituir una herramienta diferenciadora importante.

El uso de las redes sociales bajo este mismo rol es mucho más común, representa en el conglomerado una media de $57.26 \%$, pero de igual modo no es una tendencia continua a través de las plataformas; fueron los proyectos de Catarse los que levantaron este valor, su uso en Idea y Kickstarter siendo de entre el 20\% y 25\%. 
Del uso de redes, se puede distinguir tendencias fuertes en el favoritismo de ciertas páginas, el claramente preferido es Facebook que se presenta en dos tercios de las veces en las que aparece la aplicación de una red social. Esta tendencia se mantiene por las plataformas, menos en Kickstarter, donde Instagram tiene un rol dominante; esto se puede observar en la tabla 4, donde el uso de Facebook en los proyectos de Kickstarter no generó un efecto positivo significante. En el caso de Idea. me, el uso de Facebook es casi absoluto. Igualmente, el uso de Twitter e Instagram son significativos y el emprendedor les encuentra valor; pero es en el formato de Facebook donde la presentación de un proyecto es más cómoda, especialmente a la vista del empresario latino.

El uso de recompensas sigue el mismo patrón a través de las plataformas, pues si bien se distinguió un uso mayor en Idea, cuya media es siete, la media general es 4 . En cuanto se refiere a la moda, con un uso del $22.52 \%$ se concentra en cuatro recompensas, cuyo número es significativo dado que permite la suficiente complejidad a los emprendedores para ofrecer varios productos y a la vez no la hace excesivamente complicada para su ejecución.

El uso de multimedia en general es más bajo, en el uso de imágenes, por ejemplo, la moda del universo de proyectos es uno, sin embargo, esto cambia dependiendo de la plataforma, puesto que en Kickstarter es de dos y en Idea.me de cuatro; para la plataforma de Catarse las imágenes no tomaron tal importancia y una moda muy amplia se encontró en uno.

Así mismo, el favoritismo en el uso de videos y gifs es de uno, esto es entendible a partir del formato de presentación de los proyectos, que dan una importancia fundamental a un primer video, presentándolo al inicio de la página; pero los demás se relegan a la sección de la descripción junto a la demás multimedia. Su costo y mayor complejidad pueden ser los factores que hacen de esta una distribución tan inclinada y desigual, a tal punto que solo 1570 proyectos tienen más de dos videos, esto representa tan solo un 7.20\% del universo de la investigación.

La utilización de mecanismos durante la campaña, como el de las actualizaciones, es sorprendentemente bajo, su uso es muy similar en todas las plataformas, dándose mayormente en Kickstarter con $35.74 \%$, pero que reduce a $32.72 \%$ en Catarse y a $22.39 \%$ en Idea.me. Además de esto, entre quienes lo usan, la moda se encuentra en una actualización y la concentración significativamente en una, dos y tres; que sumadas representan el $17.78 \%$ de la observación. Al contrario, a quienes usan más de cinco actualizaciones, que representan tan solo el $12.47 \%$ y aquellos sobre las diez solo el $6.06 \%$.

La regresión logística binaria pudo ser desarrollada con éxito, pero se llevó a cabo bajo tres distintos modelos, debido a que, por un lado, las variables de número de recompensas, número de imágenes, número de videos y gifs, número de actualizaciones, uso de redes sociales y límite de recompensas pueden ser evaluadas para la totalidad de la muestra. Más este no es el caso para la variable número de comentarios que solo puede ser evaluada para las plataformas Kickstarter y Catarse; y para el uso de la variable botón de favoritos (Staff picked) que solo se la puede evaluar para la plataforma Kickstarter. Esto debido a la estructura de cada plataforma y las herramientas que tienen. 
Para el primer modelo, como se puede observar en la tabla 2, se evalúa el uso de las ocho variables inicialmente descritas, del total de proyectos extraídos. De tal modo se usa el modelo de nulidad $\left(X^{2}(8)=7218.68, p<.001\right)$ al cual el siguiente modelo, se adapta de forma significativamente mejor, con un $76.1 \%$ de casos clasificados correctamente, esto queriendo decir que dicho porcentaje de casos bajo el siguiente modelo serían clasificados acertadamente como exitosos y no exitosos.

El modelo también explica entre 31.4\% (Cox \& Snell $R^{2}$ ) y 41.9\% (Nagelkerke $R^{2}$ ) de varianza. Esto significa que, del volumen de proyectos exitosos, entre el $31.4 \%$ y el $41.9 \%$ de este son debido a los predictores mencionados a continuación; el resto del volumen son explicados por otros predictores no tomados en cuenta. Esto puede ser entendido como que, del universo de proyectos exitosos, un círculo que engloba entre $31.4 \%$ y $41.9 \%$ de proyectos marca el impacto que los predictores (señales) tuvieron.

\section{Tabla 2. Regresión logística binaria para estadísticas de éxito en el universo de datos}

\begin{tabular}{|l|c|c|c|c|c|c|c|c|}
\hline \multicolumn{1}{|c|}{ Predictores } & B & S.E. & Wald & df & Sig. & Exp(B) & \multicolumn{2}{c|}{$\begin{array}{c}\text { EX\% C.I.for } \\
\text { Lower }\end{array}$} \\
\hline Cuenta de Facebook & & & & & & & Upper \\
\hline Cuenta de Twitter & .145 & .042 & 12.069 & 1 & .001 & 1.156 & 1.065 & 1.255 \\
\hline Cuenta de Instagram & .451 & .042 & 113.006 & 1 & .000 & 1.570 & 1.444 & 1.706 \\
\hline Número de recompensas & .098 & .039 & 457.446 & 1 & .000 & 2.284 & 2.117 & 2.463 \\
\hline Número de imágenes & -.015 & .002 & 552.687 & 1 & .000 & 1.103 & 1.093 & 1.113 \\
\hline Número de videos y gifs & -.040 & .012 & 10.606 & 1 & .001 & .961 & .939 & .984 \\
\hline Número de actualizaciones & .287 & .006 & 2051.481 & 1 & .000 & 1.333 & 1.316 & 1.349 \\
\hline Límite de recompensas & .310 & .052 & 35.022 & 1 & .000 & 1.364 & 1.231 & 1.511 \\
\hline Constante & -2.275 & .050 & 2037.045 & 1 & .000 & .103 & & \\
\hline
\end{tabular}

Los predictores son entendidos como correctos al mantener un nivel de significancia menor a 0.05 , esto puede ser observado en la columna Sig. en la Tabla 2. Como se puede notar, todas las variables lo cumplen, aceptándose su relevancia y efecto como predictores de éxito en el lanzamiento de una campaña de crowdfunding, sea esto en un efecto positivo o negativo.

El coeficiente estándar de regresión (B) indica la dirección del efecto del predictor. Se puede notar cómo el uso de redes sociales, el número de recompensas, número de actualizaciones y límite de recompensas tienen un positivo, esto significa que mientras mayor sea el uso de estas señales, mayor la probabilidad de éxito. El número de imágenes y número de videos y gifs indican una tendencia a fallar más, mientras más de estas señales se tenga, se evidencia en sus ratios de probabilidades $\operatorname{Exp}(\mathrm{B})$, los cuales son menores a 1, lo cual indica una disminución de probabilidad de éxito. 
El coeficiente de confianza presenta ratios de probabilidad congruentes y permite aceptar las hipótesis que los demás coeficientes presentan. Esto se observa en que tanto en $\operatorname{Exp}(\mathrm{B})$ menor tanto como el mayor son ambos $\geq 1$ ó $\leq 1$.

Una segunda regresión logística fue desarrollada tan solo incluyendo los proyectos extraídos de las plataformas Kickstarter y Catarse, como se puede ver en la tabla 3, con el predictor adicional número de comentarios. Los resultados nuevamente muestran que los datos se adaptan bien al modelo $\left(X^{2}(9)=6641.47, p<.001\right)$, con un $82 \%$ de casos clasificados correctamente.

El modelo así mismo explica un Cox \& Snell $R^{2}$ igual a $29.5 \%$, mientras que tambíen se obtuvo un Nagelkerke $R^{2}$ igual a $42.2 \%$, estos como sería de esperarse, sumamente similares al primer modelo.

\section{Tabla 3. Regresión logística binaria para estadísticas de éxito en Kickstarter y Catarse}

\begin{tabular}{|c|c|c|c|c|c|c|c|c|}
\hline \multirow[t]{2}{*}{ Predictores } & \multirow[t]{2}{*}{$\mathbf{B}$} & \multirow[t]{2}{*}{ S.E. } & \multirow[t]{2}{*}{ Wald } & \multirow[t]{2}{*}{ df } & \multirow[t]{2}{*}{ Sig. } & \multirow[t]{2}{*}{$\operatorname{Exp}(B)$} & \multicolumn{2}{|c|}{$\begin{array}{l}\text { 95\% C.I.for } \\
\text { EXP(B) }\end{array}$} \\
\hline & & & & & & & Lower & Upper \\
\hline Cuenta de Facebook & .863 & .048 & 327.525 & 1 & .000 & 2.370 & 2.159 & 2.603 \\
\hline Cuenta de Twitter & .131 & .066 & 3.974 & 1 & .046 & 1.140 & 1.002 & 1.297 \\
\hline Cuenta de Instagram & .750 & .095 & 62.947 & 1 & .000 & 2.117 & 1.759 & 2.548 \\
\hline Número de recompensas & .103 & .006 & 348.305 & 1 & .000 & 1.108 & 1.096 & 1.120 \\
\hline Número de imágenes & -.006 & .003 & 4.170 & 1 & .041 & .994 & .989 & 1.000 \\
\hline Número de videos y gifs & -.002 & .012 & .026 & 1 & .872 & .998 & .974 & 1.022 \\
\hline Número de actualizaciones & .312 & .008 & 1722.780 & 1 & .000 & 1.366 & 1.346 & 1.387 \\
\hline Número de comentarios & .069 & .009 & 61.246 & 1 & .000 & 1.071 & 1.053 & 1.090 \\
\hline Límite de recompensas & .044 & .062 & .506 & 1 & .477 & 1.045 & .926 & 1.180 \\
\hline Constante & -3.692 & .113 & 1067.416 & 1 & .000 & .025 & & \\
\hline
\end{tabular}

Al evaluarse su nivel de significancia se encuentra que el número de comentarios es un predictor relevante en la consecución del éxito de las campañas, del mismo modo en el que bajo este modelo lo son el resto, menos las variables número de videos y GIF, así como el límite de recompensas.

Si se analiza puntualmente la señal comentarios, se puede observar un nivel de significancia menor a 1 que confirma su efecto predictor, un B y un $\operatorname{Exp}(\mathrm{B})$ positivos que indican el efecto predictor de éxito, y un nivel de confianza congruente que confirma la hipótesis. Por ende, mientras más uso de comentarios, mayor es la predicción de éxito.

Por último, se debió desarrollar una tercera regresión, observable en la tabla 4, para evaluar tan solo la data referente a la plataforma Kickstarter, en orden a incluir y analizar la variable Botón de favoritos (staff picked), de igual modo los datos cumplen significativamente bien con el modelo $\left(X^{2}(9)=1598.99, p<.001\right)$. 
El modelo explica también entre $43.00 \%$ (Cox \& Snell $R^{2}$ ) y 59.10\% (Nagelkerke $R^{2}$ ) de varianza. Esto significa que, del monto de proyectos exitosos, entre el $43.00 \%$ y el $59.1 \%$ de los proyectos exitosos se deben a los nueve predictores utilizados, los cuales son márgenes ligeramente mayores a los anteriores modelos.

Tabla 4. Regresión logística binaria para estadísticas de éxito en Kickstarter

\begin{tabular}{|c|c|c|c|c|c|c|c|c|}
\hline \multirow[t]{2}{*}{ Predictores } & \multirow[t]{2}{*}{$\mathbf{B}$} & \multirow[t]{2}{*}{ S.E. } & \multirow[t]{2}{*}{ Wald } & \multirow[t]{2}{*}{ df } & \multirow[t]{2}{*}{ Sig. } & \multirow[t]{2}{*}{$\operatorname{Exp}(B)$} & \multicolumn{2}{|c|}{$\begin{array}{l}\text { 95\% C.I.for } \\
\text { EXP(B) }\end{array}$} \\
\hline & & & & & & & Lower & Upper \\
\hline Cuenta de Facebook & 20.880 & 1602.290 & .000 & 1 & .990 & $\begin{array}{l}1169 \\
119607.255\end{array}$ & .000 & . \\
\hline Cuenta de Twitter & 3.465 & .871 & 15.841 & 1 & .000 & 31.986 & 5.806 & 176.227 \\
\hline Cuenta de Instagram & 3.602 & 1.032 & 12.171 & 1 & .000 & 36.656 & 4.846 & 277.247 \\
\hline Número de recompensas & .108 & .016 & 47.942 & 1 & .000 & 1.113 & 1.080 & 1.148 \\
\hline Número de imágenes & -.007 & .008 & .960 & 1 & .327 & .993 & .978 & 1.007 \\
\hline Número de videos y gifs & -.090 & .026 & 12.384 & 1 & .000 & .914 & .869 & .961 \\
\hline Número de actualizaciones & .378 & .025 & 223.360 & 1 & .000 & 1.459 & 1.388 & 1.533 \\
\hline "Staff picked" & -1.672 & .166 & 101.976 & 1 & .000 & .188 & .136 & .260 \\
\hline Límite de recompensa & -.536 & .175 & 9.334 & 1 & .002 & .585 & .415 & .825 \\
\hline Constante & -27.919 & 1602.291 & .000 & 1 & .986 & .000 & & \\
\hline
\end{tabular}

Los datos del último modelo, presente en la tabla 4, demuestran fuertes alteraciones en lo referente al uso de cuenta de Facebook, esto demuestra un nivel de significancia muy sobre 0.05 , lo cual lo hace irrelevante; el caso es similar para las otras imágenes que se presenten. Estas aparentemente no tienen mayor influencia sobre la predicción de éxito. El ser seleccionado como favorito por la plataforma, potencia radicalmente la posibilidad de éxito.

De este modo se entiende bajo estos tres modelos distintos que todas las señales evaluadas excepto el número de imágenes y número de videos y gifs, tienen fuerzas positivas para el éxito de una campaña. Estos fluctúan ligeramente dependiendo del modelo y la población evaluada. Entendiéndose a los demás efectos de éxito valores intrínsecos a la calidad del producto o a la tracción en redes.

La mayoría de los resultados encontrados se alinean a la literatura conocida, esto afianza mucho más la validez de la señalización debido a que han sido desarrollados en distintos momentos y sobre poblaciones con importantes diferencias geográficas. Los estudios de Mollick (2013) y Kunz et al. (2017) muestran sobre el universo de Kickstarter, cómo el uso de estas mismas señales comprende un predictor de éxito en las campañas. Así mismo, una observación del estudio de Kraus et al. (2016) demuestra que la señalización también es aplicable a plataformas europeas. Lo mismo puede ser replicado bajo ciertas modificaciones en otros modelos de crowdfunding como lo demuestran Ma et al. (2017); Vismara (2018) y Ahlers et al. (2015) en sus estudios sobre el crowdfunding por adquisición de acciones. Así mismo se 
encuentran ciertas distinciones, la principal es el efecto negativo que se encontró con el mayor uso de imágenes, videos y gifs, cuando el resto de la literatura evaluada indica lo contrario. Otra diferencia con la literatura, es de esperarse, es que el porcentaje de éxito es significativamente mayor en el contexto estadounidense, particularmente en Kickstarter, donde en el año de estudio de Mollick, sumaba un asombroso $48.10 \%$ y en el estudio de Kunz, años más tarde señalaría una cifra de $46.13 \%$ de éxito, presentando un nivel bastante estable, que explica que si bien el número de proyectos publicados ha seguido incrementando, la probabilidad de éxito se mantiene; mientras que el presente estudio dilucida una realidad mucho más fuerte para la escena de crowdfunding latino, donde de la extracción global, existió un 30.21\% de éxito.

\section{Conclusiones y discusión}

La escena de crowdfunding en Latinoamérica está aún en sus etapas iniciales y demuestra muchos comportamientos particulares, en consecuencia, como la aglomeración de campañas y el éxito de estas en muy pocas categorías; siempre enfocadas en productos básicos o en aquellos basados en la creación de contenidos, productos homólogos y poca variedad en recompensas que no van más allá de ligeros cambios en beneficios. Se entiende que su uso por parte del emprendedor en la mayor parte de los casos tiende a la extensión de una marca personal, sea por la de una banda de música, una editorial o autor en particular, o la creación de juegos por una agencia puntual. Esto quiere decir que la tracción del producto o servicio se genera externamente a la plataforma, y esta última es utilizada como una herramienta de pago anticipado. Esto más que como un mercado de ecommerce independiente de otros.

Se ha hallado una gran diferencia en el avance de crowdfunding dependiendo del país de origen del proyecto; esto es particularmente notable desde un inicio en la cantidad de proyectos que se han podido extraer, donde de Brasil se han generado 16 731 proyectos y en Ecuador solo 56. Claro que esto está limitado al uso de las plataformas particulares del estudio, pero de igual manera ningún país tiene una plataforma ni siquiera cercana a la magnitud de la Catarse brasileña, es más, la mayoría de los países no tiene una plataforma nacional fuerte, entendiéndose como un ejemplo claro a México (Rodríguez, 2017).

De esta diferencia en desarrollo se ha presenciado que la distribución en las categorías de uso cambia. En países con poco desarrollo se ve una concentración en categorías basadas en productos de contenido multimedia o información, en países más desarrollados se encuentra una importancia más grande en juegos digitales y productos misceláneos. Esto hace hablar mucho de la variedad de emprendedores en estos sitios y sus consumidores, así como en cierta extensión la calidad y costo de servicios de envío y la confianza que se tiene en la veracidad de los proyectos y las plataformas que los filtran.

Es importante recalcar el poco uso en general de las señales de calidad, puesto que existe en todas ellas un uso marginal, que hace que muy pocos proyectos sean aquellos que acumulan el uso de estas herramientas. En un sentido global el uso es menor; por ejemplo, el uso de página web es de $32.60 \%$, el uso de al menos una red social es $57.26 \%$, la moda en el uso de recompensas es igual a cuatro, el uso de imáge- 
nes es de una y tendiendo mucho más hacia cero, este es exactamente el mismo caso con los videos, y el uso de actualizaciones no despega de cero en un $64.85 \%$ de los casos. Es decir, existe muy poca seriedad y recursos en la mayoría de los proyectos, de modo que su factibilidad desde su concepción fue casi nula.

Esta última observación es en cierto modo positiva dado que, a pesar de haber un marginal porcentaje de éxito se ha demostrado bajo una regresión logística binaria que todas las señales presentadas a excepción del uso de imágenes, videos y gifs, generan una influencia positiva muy relevante en la probabilidad de éxito de una campaña. Esto demuestra que su uso puede predecir entre $31.4 \%$ y $41.9 \%$ del éxito de una campaña. La muestra de esfuerzo a través de estas señales genera diferencias masivas ante la poca calidad general de los proyectos. De este modo un buen uso de redes sociales, el uso de una página web para explicar el producto o servicio, la extensión de un portafolio grande, que refleja en calidad del producto y la comunicación con los consumidores en cada etapa del proyecto, muestran una gran diferencia.

Los esfuerzos que pongan las plataformas en mejorar la presentación y tracción de los proyectos de sus clientes, tanto como la natural evolución de los comercios en línea e infraestructura relacionada que surgirá en Latinoamérica, potenciarán sin duda del uso de servicios como el crowdfunding basado en recompensa. Este es el inicio de un largo camino que sólo se acelerará con el desarrollo de estrategias para cautivar a un muy receloso mercado, que, sin embargo, es muy grande e incrementalmente adepto a las transacciones en línea y a la influencia de países más desarrollados en este sentido.

\section{Referencias}

Agrawal, AK, Catalini, C., \& Goldfarb, A. (2013). Some Simple Economics of Crowdfunding. Nber Working Paper Series. Recuperado de: https://bit.ly/2w8KWdg

Ahlers, Gerrit K.C., \& Cumming, D., Günther, C., \& Schweiser, D. (2015). Signaling in Equity Crowdfunding. Entrepreneurship: Theory and Practice, 955-980. https://doi.org/10.1111/ etap. 12157

Banco Mundial (2013). Crowdfunding's Potential for the Developing World. Finance and Private Sector Development Department, 1-102.

Belleflamme, P., Lambert, T., \& Schwienbacher, A. (2012). Crowdfunding: Tapping the right crowd. Journal of Business Venturing, 29(5), 585-609. https://doi.org/10.1016/j.jbusvent.2013.07.003

Berger, A. N., \& Udell, G. F. (1995). Relationship Lending and Lines of Credit in Small Firm Finance. The Journal of Business, 68(3), 351. https://doi.org/10.1086/296668

Canela, M., Lora, M., \& Estrella. (2011). Cómo hacer una Regresión Logística binaria "paso a paso" (II): análisis multivariante. Docuweb FABIS, 34.

Cardon, M., Sudek, R., \& Mitteness, C. (2009). The impact of perceived entrepreneurial passion on angel investing. Frontiers of Entrepreneurship Research 29. Recuperado de: https://bit. ly/3d6p57k

Connelly, B. L., Certo, S. T., Ireland, R. D., \& Reutzel, C. R. (2011). Signaling Theory: A Review and Assessment. Journal of Management. Recuperado de: https://bit.ly/3bo1VbH

Courtney, C., Dutta, S., \& Li, Y. (2017). Resolving Information Asymmetry: Signaling, Endorsement, and Crowdfunding Success. Entrepreneurship: Theory and Practice, 41(2), 265-290. https://doi.org/10.1111/etap.12267 
Chakraborty, S., \& Swinney, R. (2016). Signaling to the Crowd: Private Quality Information and Rewards-Based Crowdfunding. Ssrn. https://doi.org/10.2139/ssrn.2885457

Davies, W. E., \& Giovannetti, E. (2018). Signalling experience \& reciprocity to temper asymmetric information in crowdfunding evidence from 10,000 projects. Technological Forecasting and Social Change, (March), 1-14. https://doi.org/10.1016/j.techfore.2018.03.011

Dehling, S. (2013). Crowdfunding - A Multifaceted Phenomenon. University of Twente. Recuperado de: https://bit.ly/37ehkb7

Etter, V., Grossglauser, M., \& Thiran, P. (2013). Launch hard or go home! Proceedings of the First ACM Conference on Online Social Networks-COSN '13, 177-182. https://doi. org/10.1145/2512938.2512957

Jiang, Z., \& Benbasat, I. (2007). Investigating the influence of the functional mechanisms of online product presentations. Information Systems Research, 18(4), 454-470.

Kickante (2018). Os 10 maiores sites de Crowdfunding no Brasil. Recuperado de: https://bit. ly/33hfRAH

Kim, P. H., Buffart, M., \& Croidieu, G. (2016). TMI: Signaling Credible Claims in Crowdfunding Campaign Narratives. Group and Organization Management, 41(6), 717-750. https://doi. org/10.1177/1059601116651181

Kickstarter (2018) Estadísticas. Recuperado de: https://bit.ly/37QZcpq (2018-09-10).

Kunz, M. M., Bretschneider, U., Erler, M., \& Leimeister, J. M. (2017). An empirical investigation of signaling in reward-based crowdfunding. Electronic Commerce Research (Vol. 17). https://doi.org/10.1007/s10660-016-9249-0

Kraus, S., Richter, C., Brem, A., Cheng, C.-F., \& Chang, M.-L. (2016). Strategies for reward-based crowdfunding campaigns. Journal of Innovation \& Knowledge, 1(1), 13-23. https://doi. org/10.1016/j.jik.2016.01.010

Lambert, T., \& Schwienbacher, A. (2010). An Empirical Analysis of Crowdfunding. SSRN Working Paper. Recuperado de: https://bit.ly/2OIBQKs

Lynn, M. (1991). Scarcity effects on value: A quantitative review of the commodity theory literature. Psychology \& Marketing, 8(1), 43-57. https://doi.org/10.1002/mar.4220080105

Mason, C. M., \& Harrison, R. T. (2004). Improving access to early stage venture capital in regional economies: A new approach to investment readiness. Local Economy, 19(2), 159-173.

Ma, X., Yang, M., Li, Y., \& Zhang, J. (2017). Signaling factors in overfunding: An empirical study based on Crowdcube. 14th International Conference on Services Systems and Services Management, ICSSSM 2017 - Proceedings, (2016). https://doi.org/10.1109/ ICSSSM.2017.7996208

Massolution (2015). The crowdfunding industry report. Recuperado de: https://bit.ly/39wqn97

Martens, M. L., Jennings, J. E., \& Jennings, P. D. (2007). Do the stories they tell get them the money they need? The role of entrepreneurial narratives in resource acquisition. Academy of Management Journal, 50(5), 1107-1132.

Mollick, E. (2013). The dynamics of crowdfunding: An exploratory study. Journal of Business Venturing, 29(1), 1-16. https://doi.org/10.1016/j.jbusvent.2013.06.005

Nahapiet, J., \& Ghoshal, S. (1998). Social Capital, Intellectual Capital, and the Organizational Advantage. The Academy of Management Review, 23(2), 242. https://doi.org/10.2307/259373

Rentería, C. (2016). Las plataformas de crowdfunding en América Latina. Nueva York. https://doi. org/10.13140/RG.2.2.29576.01286/1

Rodríguez, R. (2017). Evolución del ecosistema de crowdfunding en México 2015-2017. Crowdfunding México-BIMCON.

Rojas, L. (2017). Situación del financiamiento a pymes y empresas nuevas en América Latina. CAF. Santiago. Primera Edición. Recuperado de: https://bit.ly/38jSXKD

Spence, M. (1973). Job market signaling. The Quarterly Journal of Economics, 87, 355-374. doi: $10.2307 / 1882010$ 
Tranmer, M., \& Elliot, M. (2005). Binary Logistic Regression. Cathie Marsh Centre for Census and Survey Research, (1), 3-43. https://doi.org/10.4135/9781412995627

Thelwall, M. (2001). A web crawler design for data mining. Journal of Information Science, 27(5), 319-325. https://doi.org/10.1177/016555150102700503

Vismara, S. (2018). Signaling to Overcome Inefficiencies in Crowdfunding Markets. The Economics of Crowdfunding. https://doi.org/https://doi.org/10.1007/978-3-319-66119-3_3

Walsh, A. (1987). Teaching Understanding and Interpretation of Logit Regression. Teaching Sociology, 15(2), 178-183. https://doi.org/10.2307/1318033

Wells, J. D., Valacich, J. S., \& Hess, T. J. (2011). What signal are you sending? How website quality influences perceptions of product quality and purchase intentions. MIS Quarterly, 35(2), 373-396. 